\title{
Identification of NRPS gene families from Neotyphodium/Epichloë endophytic fungi that form mutualistic associations with cool season grasses.
}

\author{
R. D. JOHNSON, C. R. VOISEY, J. M. PRATT, L. J. JOHNSON and G. T BRYAN \\ AgResearch Grasslands, Private Bag 11008, Palmerston North, New Zealand
} richard.johnson@agresearch.co.nz

\begin{abstract}
Neotyphodium and Epichloë species are closely related asexual and sexual endophytic fungi, respectively, that form symbiotic associations with cool season grasses of the sub family Pooideae, including several important forage and turf grass species. The endophytes confer a number of advantages to their hosts, but also can cause animal toxicosis. These positive and negative effects are, in many cases, due to the production of fungal secondary metabolites. In filamentous fungi secondary metabolite genes are commonly clustered and are well documented to often contain non-ribosomal peptide synthetases (NRPSs) at their core. Members of this gene family encode large multifunctional proteins that synthesise a diverse range of bioactive compounds, many of which have been shown to serve as pathogenicity or virulence factors, in addition to suggested roles in niche adaptation. We have used a degenerate PCR approach to identify members of the NRPS gene family from Neotyphodium and Epichloë species, and have shown that at least 13 NRPS genes exist among these genomes. The distribution of these genes among different Neotyphodium/Epichloë lineages suggests that a common ancestor contributed most of the complement of NRPS genes, which have been either retained or lost during the evolution of these fungi.
\end{abstract}

Keywords: Neotyphodium, Epichloë, non-ribosomal peptide synthetase, NRPS

\section{Introduction}

Temperate grasses belonging to the family Poodieae commonly harbour fungal endophytes of the Neotyphodium/Epichloë genus. For example, $N$. lolii and $N$. coenophialum are fungal endophytes that live entirely within the intercellular spaces of perennial ryegrass and tall fescue, respectively. Infection is symptomless and the endophytes rely entirely on the host plant for dissemination via seed or through vegetative structures (see review by Schardl et al. 2004). The association is mutually beneficial since the endophyte confers a number of biotic and abiotic advantages to the host (Scott 2001; Schardl et al. 2004). Some of these host benefits are due to the production of fungal secondary metabolites such as peramine (pyrrolopyrazine), loline (aminopyrrolizidine), ergovaline (ergopeptine) and lolitrem (indole diterpene) alkaloids (Tanaka et al. 2005; Spiering et al. 2005; Panaccione et al. 2001; Young et al. 2006), which can also cause mammalian toxicoses in some cases.

In filamentous fungi secondary metabolite genes are commonly clustered (Keller \& Hohn 1996) and are well documented to often contain non-ribosomal peptide synthetases (NRPSs) at their core (Walton 2006; Gardiner et al. 2004). Members of this gene family encode large multifunctional proteins (Finking \& Marahiel 2004) that synthesise a diverse range of bioactive compounds, some of which serve as pathogenicity or virulence factors (Walton 2006; Johnson et al. 2000; Gardiner et al. 2004; Lee et al. 2005), in addition to suggested roles in niche adaption (Lee et al. 2005). In this study we sought to survey the numbers of NRPS genes from endophytes of the Neotyphodium/Epichloe genus. Two NRPS genes have already been shown to play important roles in this symbiosis; peramine and ergovaline are synthesised wholly or in part by NRPSs (Panaccione et al. 2001; Tanaka et al. 2005). In addition, we carried out a comprehensive strain distribution study across different taxonomic groupings of endophytes (Tsai et al. 1994; Moon et al. 2004), including those of non-hybrid, dihybrid or tri-hybrid origin to gain an insight into the evolution of these genes within the Neotyphodium/Epichloë complex.

Degenerate PCR with primers designed to conserved motifs within NRPS genes, have been shown to be useful for identifiying members of this gene family from a number of filamentous fungi (Pannaccione 1996; Johnson et al. 2000). We used this approach in the present study to identify both conserved adenylation domains, characteristic of all classes of NRPSs, as well as methyl transferase domains, which are restricted to certain classes of NRPSs (Finking \& Marahiel 2004) and are responsible for Nmethylation of the peptide product.

\section{Materials and Methods}

\section{Endophyte strains}

Endophyte strains used in this study are listed in Table 2.

\section{Genomic DNA}

Genomic DNA was kindly provided by Andrew Griffiths (AgResearch Grasslands, New Zealand) and Professor Christopher Schardl (University of Kentucky, USA). DNAs were subsequently amplified using Genomiphi (Amersham) as per the manufacturer's instructions, and diluted to $100 \mu \mathrm{g}$ per $\mathrm{ml}$ prior to use in PCR.

\section{Degenerate PCR and cloning of NRPS fragments}

Primers were designed to both conserved adenylation and methyl transferase domains as follows. The forward and reverse primers for the adenylation domain were designed to the conserved motifs Y G P T E and Y K T G D L, respectively as described in Johnson et al. (2000). For the methyl transferase primers, core motifs N S V A/V Q Y F P and I/V K/E/Q H V E V/I L/I P K were used to design forward and reverse primers respectively. Primer pairs were used in degenerate PCR experiments, with genomic DNA of $N$. lolii strains Lp19 and AR66, E. typhina strain E8, and taxonomic group FATG-3 strain AR501, as described in Johnson et al. (2000). PCR products were ligated into the pCRTOPO2.1 TA cloning vector (Invitrogen) and clones were sequenced using an M13R universal primer and the BigDye Terminator v3.1 Cycle Sequencing Kit (Applied Biosystems). Sequences were run on an Applied Biosystems automated ABI3100 sequencer.

\section{Bioinfomatics}

Sequences were manually trimmed of vector sequence and degenerate primer binding sites were identified and removed. Sequence alignments were perfomed using AlignX from the vector NTI suite 9.0 (Invitrogen) and a dendrogram was constructed showing the relatedness between NRPS sequences. 
Table 1 Classification of NRPS genes identified from endophytes of the Neotyphodium/Epichloë complex.

\begin{tabular}{lllll}
\hline NRPS & e-value & Top hit accession & Proposed function & Reference \\
\hline 1 & $2 e-38$ & AAA74270 & Toxin -like & This Study \\
2 & $2 e-39$ & BAE06841 & Siderophore & This Study \\
3 & $7 e-16$ & BAE06838 & Unknown & This Study \\
4 & $2 e-29$ & BAE06845 & Unknown & This Study \\
5 & $2 e-22$ & BAE06845 & Unknown & This Study \\
6 & $1 \mathrm{e}-16$ & XP_682495 & Methylated peptide & This Study \\
7 & $2 e-25$ & AAX09985 & Methylated peptide & This Study \\
8 (PerA) & $8 e-49$ & BAE06845 & Peramine synthesis & This Study; Tanaka et al. (2005) \\
9 & $2 e-33$ & XP_385548 & Siderophore & This Study \\
CPS1 & $3 e-41$ & AAP12366 & Virulence (CPS1-like) & This Study \\
LpsA & 0 & AAL26315 & Ergovaline synthesis & Panaccione et al. (2002) \\
LpsB & 0 & CAD28788 & Ergovaline synthesis & Fleetwood et al.(2007) \\
AC202 & 0 & AAA74265 & Unknown & Panaccione (1996) \\
AC203 & 0 & AAA74268 & Unknown & Panaccione (1996) \\
\hline
\end{tabular}

Table 2 Pattern of distribution of NRPS genes across the Neotyphodium/Epich/oë complex.

\begin{tabular}{|c|c|c|c|c|c|c|c|c|c|c|c|c|c|c|c|c|c|}
\hline \multirow[t]{2}{*}{ Strain } & \multirow[t]{2}{*}{ Species $^{1}$} & \multirow[t]{2}{*}{$\begin{array}{c}\text { Hybrid } \\
\text { components }\end{array}$} & \multicolumn{8}{|c|}{ 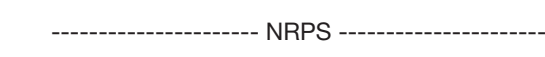 } & \multicolumn{2}{|r|}{ CPS } & \multicolumn{2}{|c|}{ AC20 } & \multicolumn{2}{|c|}{ Lps } & \multirow[b]{2}{*}{ Tef } \\
\hline & & & 1 & 2 & 3 & 4 & 5 & 6 & 7 & 8 & 9 & 1 & 3 & 2 & A & B & \\
\hline AR512 & N.coen. & Ef $\times$ Et $\times$ Eb & + & + & - & - & + & - & - & + & + & + & + & + & + & + & + \\
\hline AR535 & N.coen. & Ef $x$ Et $x$ Eb & + & + & - & - & + & - & - & + & + & + & + & + & + & + & + \\
\hline AR514 & N.coen. & Ef $x$ Et $x$ Eb & + & + & - & - & + & - & - & + & + & + & + & + & + & + & + \\
\hline AR525 & N.coen. & Ef $x$ Et $x$ Eb & + & + & - & - & + & - & - & + & + & + & + & + & + & + & + \\
\hline AR546 & N.coen. & Ef $x$ Et $x$ Eb & + & + & + & - & + & - & - & + & + & + & + & + & + & + & + \\
\hline AR567 & N.coen. & Ef $x$ Et $x$ Eb & + & + & + & - & + & - & - & + & + & + & + & + & + & + & + \\
\hline $\mathrm{RS} 2$ & N.coen. & Ef $x$ Et $x$ Eb & + & + & + & - & + & - & - & + & + & + & + & + & + & + & + \\
\hline RS6 & N.coen. & Ef $x$ Et $x$ Eb & + & + & + & - & + & - & - & + & + & + & + & + & + & + & + \\
\hline AR604 & N.coen. & Ef $x$ Et $x$ Eb & + & + & + & - & + & - & - & + & + & + & + & + & + & + & + \\
\hline AR542 & N.coen. & Ef $x$ Et $x$ Eb & + & + & + & - & + & - & - & + & + & + & + & + & - & - & + \\
\hline AR584 & N.coen. & Ef $x$ Et $x$ Eb & + & + & + & - & + & - & - & + & + & + & + & + & - & - & + \\
\hline AR593 & N.coen. & Ef $x$ Et $x$ Eb & + & + & + & - & + & - & - & + & + & + & + & + & - & - & + \\
\hline AR565 & N.coen & Ef $x$ Et $x$ Eb & + & + & + & - & + & - & - & + & + & + & + & + & + & + & + \\
\hline AR553 & FaTG-2 & Ef x Eb & + & + & - & - & + & - & - & + & + & + & + & - & + & + & + \\
\hline AR555 & FaTG-2 & $\mathrm{Ef} \times \mathrm{Eb}$ & + & + & - & - & + & + & - & + & + & + & + & - & + & + & + \\
\hline AR501 & FaTG-3 & Et $x$ Eb & + & + & - & - & + & + & + & + & + & + & + & + & - & - & + \\
\hline AR502 & FaTG-3 & Et $x$ Eb & + & + & - & + & + & + & + & + & + & + & + & + & - & - & + \\
\hline AR506 & FaTG-3 & Et $x$ Eb & + & + & - & - & + & + & + & + & + & + & + & - & - & - & + \\
\hline AR507 & FaTG-3 & Et $x$ Eb & + & + & - & - & + & + & + & + & + & + & + & + & - & - & + \\
\hline AR510 & FaTG-3 & Et $x$ Eb & + & + & - & - & + & + & + & + & + & + & + & + & - & - & + \\
\hline AR26 & LpTG-2 & Et $x$ NI & + & + & - & + & + & + & + & + & + & + & + & + & + & + & + \\
\hline Lp1 & LpTG-2 & $\mathrm{Et} \times \mathrm{NI}$ & + & + & - & + & + & + & + & + & + & + & + & + & + & + & + \\
\hline E938 & N.aust. & Et $x$ Ef & + & + & - & + & + & + & + & + & + & + & + & + & + & + & + \\
\hline E1169 & N.tem. & Et $x$ Ef & + & + & - & + & + & + & + & + & + & + & + & + & - & - & + \\
\hline Fp1 & N.unc. & Et $x$ Ebr & + & + & - & + & + & + & - & + & + & + & + & + & - & - & + \\
\hline AR1002 & N.unc. & Et $x$ Ebr & + & + & - & + & + & + & - & + & + & + & + & + & - & - & + \\
\hline Fp3 & N.unc. & Et $x$ Ebr & + & + & - & + & + & + & - & + & + & + & + & + & - & - & + \\
\hline Fp5 & N.unc. & Et $x$ Ebr & + & + & - & + & + & + & - & + & + & + & + & + & - & - & + \\
\hline AR1007 & $N$ sieg. & Ef $x$ Ebr & + & + & - & + & + & + & - & + & + & + & + & + & - & - & + \\
\hline E915 & N.sieg. & Ef $x$ Ebr & + & + & + & + & + & + & - & + & + & + & + & + & - & - & + \\
\hline E822 & N. mel. & $\mathrm{Ef} \times \mathrm{Na}$ & + & + & - & + & + & + & + & + & + & + & + & + & + & + & + \\
\hline AR1501 & E. fes. & Non-hybrid & + & + & - & - & + & - & - & + & + & + & + & - & + & + & + \\
\hline Frr1 & E. fes. & Non-hybrid & + & + & + & - & + & - & + & + & + & + & + & - & + & + & + \\
\hline AR40 & N. Iolii & Non-hybrid & + & + & - & - & + & - & - & + & + & + & + & - & - & - & + \\
\hline
\end{tabular}




\begin{tabular}{|c|c|c|c|c|c|c|c|c|c|c|c|c|c|c|c|c|c|}
\hline \multirow[t]{2}{*}{ Strain } & \multirow[t]{2}{*}{ Species $^{1}$} & \multirow{2}{*}{$\begin{array}{c}\text { Hybrid } \\
\text { components }\end{array}$} & \multicolumn{8}{|c|}{----------------------- NRPS ---------------------- } & \multicolumn{2}{|r|}{ CPS } & \multicolumn{2}{|c|}{ AC20 } & \multicolumn{2}{|c|}{ Lps } & \multirow[b]{2}{*}{ Tef } \\
\hline & & & 1 & 2 & 3 & 4 & 5 & 6 & 7 & 8 & 9 & 1 & 3 & 2 & A & $\mathrm{B}$ & \\
\hline AR1 & N. Iolii & Non-hybrid & + & + & - & - & + & + & - & + & + & + & + & - & + & + & + \\
\hline AR12 & N. Iolii & Non-hybrid & + & + & - & - & + & + & - & + & + & + & + & - & + & + & + \\
\hline AR56 & N. Iolii & Non-hybrid & + & + & - & - & + & + & - & + & + & + & + & - & + & + & + \\
\hline AR59 & N. Iolii & Non-hybrid & + & + & - & - & + & + & - & + & + & + & + & - & + & + & + \\
\hline AR29 & N. Iolii & Non-hybrid & + & + & - & - & + & + & - & + & + & + & + & - & + & + & + \\
\hline AR30 & N. Iolii & Non-hybrid & + & + & - & - & + & + & - & + & + & + & + & - & + & + & + \\
\hline AR42 & N. Iolii & Non-hybrid & + & + & + & - & + & + & - & + & + & + & + & - & + & + & + \\
\hline AR47 & N. Iolii & Non-hybrid & + & + & + & - & + & + & + & + & + & + & + & - & - & - & + \\
\hline AR48 & N. Iolii & Non-hybrid & + & + & + & - & + & + & + & + & + & + & + & - & - & - & + \\
\hline AR49 & N. Iolii & Non-hybrid & + & + & + & - & + & + & + & + & + & + & + & - & - & - & + \\
\hline AR64 & N. Iolii & Non-hybrid & + & + & + & + & + & + & + & + & + & + & + & - & - & - & + \\
\hline AR17 & N. Iolii & Non-hybrid & + & + & + & + & + & + & + & + & + & + & + & + & - & - & + \\
\hline AR66 & N. Iolii & Non-hybrid & + & + & + & + & + & + & + & + & + & + & + & + & - & - & + \\
\hline E8 & E. typh. & Non-hybrid & + & + & - & + & + & + & - & + & + & + & + & + & - & - & + \\
\hline E505 & E. typh. & Non-hybrid & + & + & - & + & + & + & - & + & + & + & + & + & - & - & + \\
\hline- & N. aot. & Non-hybrid & + & + & - & + & + & + & - & + & + & + & - & + & - & - & + \\
\hline E354 & E. sylv. & Non-hybrid & + & + & - & + & + & + & - & + & + & + & + & + & - & - & + \\
\hline E503 & E. sylv. & Non-hybrid & + & + & - & + & + & + & - & + & + & + & + & + & - & - & + \\
\hline E422 & E. clark. & Non-hybrid & + & + & - & + & + & - & - & + & + & + & + & + & + & + & + \\
\hline E2772 & E.gl. & Non-hybrid & + & + & - & + & + & - & - & + & + & + & + & - & + & + & + \\
\hline E799 & E.br. & Non-hybrid & + & + & - & + & + & + & - & + & + & + & + & + & - & - & + \\
\hline E501 & E. br. & Non-hybrid & + & + & - & + & + & + & - & + & + & + & + & + & + & + & + \\
\hline E248 & E.ba & Non-hybrid & + & + & - & + & + & + & - & + & + & + & + & - & + & + & + \\
\hline E1031 & E.ba. & Non-hybrid & + & + & - & + & + & + & - & + & + & + & + & - & - & - & + \\
\hline E57 & E.am. & Non-hybrid & + & + & - & + & + & + & - & + & + & + & + & - & + & + & + \\
\hline E52 & E.am. & Non-hybrid & + & + & - & + & + & + & + & + & + & + & + & - & - & - & + \\
\hline E1040 & E.b. & Non-hybrid & + & + & - & + & + & + & - & + & + & + & + & - & + & + & + \\
\hline
\end{tabular}

${ }^{1}$ N.coen.- N. coenophialum; N.aust.-N. australiense; N.tem.-N. temblederae; N.unc.- N. uncinatum; N.sieg. - N. siegelii; N.mel.- N. melicola; E. fes.-E. festucae; E.typh.-E. typhina; N. aot.-N. aotearoae; E. sylv.-E.sylvatica; E.clark.-E. clarkii; E.gl. - E. glyceriae; E.br.-E. bromicola; E.ba.- E. baconii; E.a- E. amirillans; E.b.- E. brachyeltri

\section{Strain distribution studies}

Gene specific primers were designed to NRPS related sequences identified in this study, to a further NRPS gene (here designated NRPS9) identified from an N. lolii EST library (Johnson et al. 2007), to additional sequences previously published (Panaccione 1996; Panaccione et al. 2001) and to $L p s B$, a second NRPS identified in the ergovaline biosynthetic gene cluster (Fleetwood et al. 2007). These primers were used in PCR experiments with different endophyte strains to determine the distribution of the different NRPS sequences across the Neotyphodium/Epichloë complex. Primers designed to the conserved translation elongation factor gene were used to verify the integrity of the DNA in PCR experiments. Primer pairs were used in PCR experiments using standard conditions.

\section{Results and Discussion}

Cloning, sequencing and bioinfomatics of NRPS fragments

Degenerate PCR, using primers designed to either adenylation (A) or methyl transferase (MT) domain motifs, both yielded products of the target size, which ranged between $250 \mathrm{bp}$ and $350 \mathrm{bp}$ (data not shown). After cloning, 18 A domain and 6 MT domain NRPS fragments were sequenced for each of the four strains used in the degenerate PCR experiments. Sequence alignment revealed that the NRPS sequences fell into eight distinct clades (Figs. 1A \& 1B). NRPS sequences that fell within the same clade differed by the presence of species specific SNPs, which in some instances corresponded to multiple copies of the gene within hybrid genomes. A further unique sequence was also identified from the degenerate PCR screen which had homology to a related adenylate forming enzyme, but is not strictly an NRPS. Due to the multimodular nature of NRPS genes, probes corresponding to NRPSs representative of the different clades were used to screen a $N$. lolii BAC library. For NRPSs represented in this library, hybridisation to different BAC clones indicated that the NRPS fragments most likely represented independent NRPS genes (data not shown) rather than components of the same gene. Because some NRPS sequences were specific to strains other than Lp19, not all of them were represented in the BAC library. However, classification based on homology to other reported NRPS sequences and their strain distribution in the present study provided evidence that they were likely to be independent of each other.

\section{NRPS classification}

Based on BLASTX analysis against the non redundant GenBank databases and the classifications of Lee et al. (2005), we determined that the NRPS gene fragments identified in this study corresponded to several different classes (Table 1). These include NRPSs involved in the biosynthesis of siderophores, toxins involved in pathogenesis, $\mathrm{N}$-methylated peptides, and several that could not be classified. In addition we also identified a degenerate PCR sequence with homology to CPS1 (Lu et al. 2003), that 
Figure 1A Relationship between NRPS adenylation domains cloned by degenerate PCR

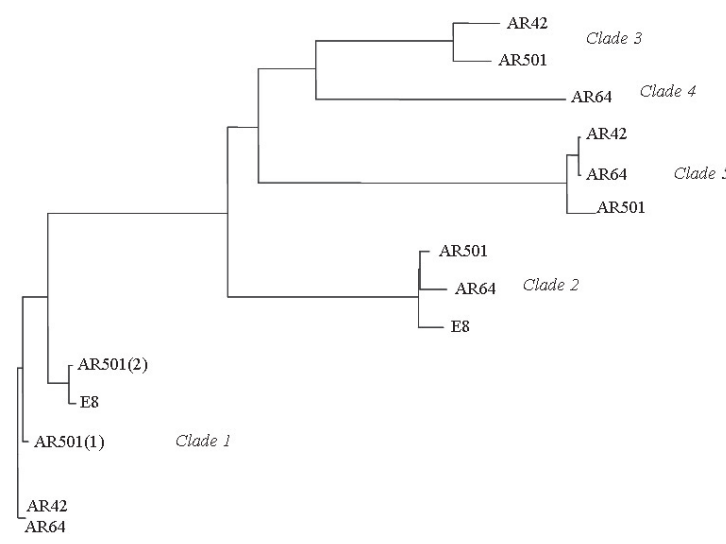

Figure 1B Relationship between NRPS methyl transferase domains cloned by degenerate PCR

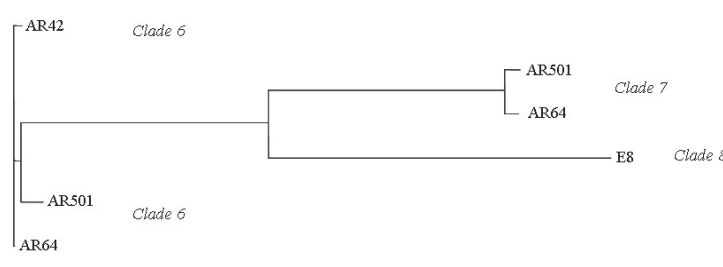

whilst not strictly an NRPS has a proven role in pathogenicity across a wide range of fungal genera. Using primers designed specifically to methyl transferase domains, a feature present only in some NRPS systems, we were able to identify three NRPSs related to this class. One of these, NRPS8, matched the sequence for the methyl transferase domain of perA, a NRPS shown to be required for peramine biosynthesis in Epichloë festucae (Tanaka et al. 2005). A further NRPS sequence (NRPS9) was independently identified from an $N$. lolii EST library (Johnson et al. 2007). Additional endophyte NRPS genes that have been previously reported but not identified in the present study were lpsA (Panaccione et al. 2001), and LpsB (Fleetwood et al. 2007) which are both involved in ergovaline biosynthesis, as well as AC203 and AC202 (Panaccione 1996), NRPSs with unknown function. Some of the NRPS gene fragments reported here matched degenerate PCR products cloned in other studies, such as NRPS2 which matches ps4 (Tanaka et al. 2005), NRPS1 which matches AC205 and ps7 (Panaccione 1996; Tanaka et al. 2005), and NRPS8 which matches AC406 and perA (Panaccione 1996; Tanaka et al. 2005).

\section{Strain distribution study}

Results of a PCR based strain distribution study are presented in Table 2. Some NRPS genes (1, 2, 5, 8, 9, AC203), as well as a sequence related to CPS1, showed a global pattern of distribution indicating they have been strongly conserved during the evolution of these fungi. Apart from NRPS8 (perA) which has been shown to be required for the synthesis of the anti-insect feeding deterrent peramine (Tanaka et al. 2005) the functions of the other conserved NRPSs identified in this study have not previously been assigned. However, their global retention within the genomes of Neotyphodium/Epichloë species suggests they have been strongly selected for during symbiosis of these endophytes with their grass hosts and presumably play a role in these associations. We have now shown that NRPS2 and NRPS4 are involved in the biosynthesis of siderophores - essential, low molecular weight, iron-chelating agents (Johnson et al. 2007). For the remainder of the NRPS genes (3, 6, 7, 4, AC202, lpsB and $\operatorname{lps} A$ ), their distribution across the strain collection was inconsistent. These NRPS genes do not therefore have essential roles in these fungi. The absence of some NRPS genes from certain endophyte species is most likely to be due to gene loss through speciation or hybridisation. It is likely that NRPS genes with essential functions such as NRPS2, involved in siderophore biosynthesis, have been globally retained, whilst others with noncritical function have been lost from some strains. Interestingly, the $N$. coenophialum strains have equal or lower numbers of NRPS genes compared to non-hybrid species, despite the fact that they are tri-hybrid in origin. For example, NRPS4, NRPS6 and NRPS7 are all absent in N. coenophialum despite these genes being present in their component genomes. This strongly suggests that NRPS loss is commonly associated with hybridisation events between different endophyte lineages. An alternative hypothesis for the discontinuous distribution of some NRPS genes is that they were not present in a common ancestor and so instead of being lost during the evolution of these fungi they were introduced into multiple species through horizontal gene transfer (Walton 2000). However, given the numerous instances that this would need to occur, and the lack of strong evidence for such events in filamentous fungi, this scenario seems unlikely.

\section{Conclusions}

Our results indicate that Neotyphodium/Epichloë species contain at least 13 NRPS genes, including members (for example, NRPS2) that are conserved within the ascomycetes, as well as some that appear to be unique to these grass endophytes. This suggests that Neotyphodium/Epichloë species are likely to synthesise a much greater number of small peptide secondary metabolites than has currently been identified. Further studies are currently in progress to functionally characterise the additional novel NRPS genes identified in this study with the aim to identify possible roles during Neotyphodium/Epichloë symbioses with their grass hosts, as well as to discover the potential secondary metabolite end products.

\section{ACKNOWLEDGEMENTS}

We acknowledge the Foundation for Research Science and Technology New Zealand (contract C10X0203) and AgResearch AR\&C (contract 9958/1) for funding.

\section{References}

Finking, R.; Marahiel, M.A. 2004. Biosynthesis of nonribosomal peptides1. Annual Review of Microbiology 58: 453-88.

Fleetwood, D.J.; Scott, B.; Lane, A.; Tanaka, A.; Johnson, R.D. 2007. A complex ergovaline gene cluster in Epichloë endophytes of grasses. Applied Environmental Microbiology: in press.

Gardiner, D.M.; Cozijnsen, A.J.; Wilson, L.M.; Pedras, M.S.; Howlett, B.J. 2004. The sirodesmin biosynthetic gene cluster of the plant pathogenic fungus Leptosphaeria maculans. Molecular Microbiology 53: 1307-1318.

Johnson, R.D.; Johnson, L; Itoh, Y.; Kodama, M.; Otani, H.; Kohmoto, K. 2000. Cloning and characterization of a cyclic peptide synthetase gene from Alternaria alternata apple 
pathotype whose product is involved in AM-toxin synthesis and pathogenicity. Molecular Plant Microbe Interactions 13: 742-753.

Johnson, R.D., Khan, A.K., Voisey, C.R., Bassett, S., Gaborit C., Johnson, L.J., Christensen, M., McCulloch, A., Bryan, G.T. 2007. Analysis of expressed sequence tags derived from the endophytic fungus Neotyphodium lolii grown in vitro and in association with its host plant perennial ryegrass. pp. $501 \mathrm{In}$ : Proceedings of the 6th International Symposium on Fungal Endophytes of Grasses. Grassland Research and Practice Series No 13. New Zealand Grassland Association.

Keller, N.P.; Hohn, T.M. 1996. Metabolic pathway gene clusters in filamentous fungi.Fungal Genetics and Biology 21: 17-29.

Lee, B-N.; Kroken, S.; Chou, D.Y.T.; Robbertse, B.;Yoder, O.C.; Turgeon, B.G. 2005. Functional analysis of all nonribosomal peptide synthetases in Cochliobolus heterostrophus reveals a factor, NPS6, involved in virulence and resistance to oxidative stress. Eukaryotic Cell 4: 545-555.

Lu, S.W.; Kroken, S.; Lee, B.N.; Robbertse, B.; Churchill, A.C.; Yoder, O.C.; Turgeon, B.G. 2003. A novel class of gene controlling virulence in plant pathogenic ascomycete fungi. Proceedings of the National Academy of Science. USA 100: 5980-5985.

Moon, C.D.; Craven, K.D.; Leuchtmann, A.; Clement, S.L.; Schardl, C.L. 2004. Prevalence of interspecific hybrids amongst asexual fungal endophytes of grasses. Molecular Ecology 13: 1455-1467.

Panaccione, D.G. 1996. Multiple families of peptide synthetase genes from ergopeptine-producing fungi. Mycological Research 100: 429-436.

Panaccione, D.G., Scott-Craig, J.S., Pocard, J.A., Walton, J.D. 1992. A cyclic peptide synthetase gene required for pathogenicity of the fungus Cochliobolus carbonum on maize. Proceedings of the National Academy of Science U S
A. 89: 6590-6594.

Panaccione, D.G.; Johnson, R.D.; Wang, J.; Young, C.A.; Damrongkool, P.; Scott, B.; Schardl, C.L. 2001. Elimination of ergovaline from a grass-Neotyphodium endophyte symbiosis by genetic modification of the endophyte. Proceedings of the National Academy of Science U S A. 98: 12820-12825.

Schardl, C.L.; Leuchtmann, A.; Spiering, M.J. 2004. Symbioses of grasses with seedborne fungal endophytes. Annual Review of Plant Biology 55: 315-340.

Scott, B. 2001. Epichloë endophytes: fungal symbionts of grasses. Current Opinion in Microbiology 4: 393-398.

Spiering, M.J.; Moon, C.D.; Wilkinson, H.H.; Schardl, C.L. 2005. Gene clusters for insecticidal loline alkaloids in the grass-endophytic fungus Neotyphodium uncinatum. Genetics 169: 1403-1414.

Tanaka, A.; Tapper, B.A.; Popay, A.; Parker, E.J.; Scott, B. 2005. A symbiosis expressed non-ribosomal peptide synthetase from a mutualistic fungal endophyte of perennial ryegrass confers protection to the symbiotum from insect herbivory. Molecular Microbiology 57: 1036-1050.

Tsai, H.F.; Liu, J.S.; Staben, C.; Christensen, M.J.; Latch, G.C.M.; Siegel, M.R.; Schardl, C.L. 1994. Evolutionary diversification of fungal endophytes of tall fescue grass by hybridization with Epichloë species. Proceedings of the National Academy of Science US A. 91: 2542-2546.

Walton, J.D. 2000. Horizontal gene transfer and the evolution of secondary metabolite gene clusters in fungi: an hypothesis. Fungal Genetics and Biology 30: 167-171.

Walton, J.D., 2006. HC-toxin. Phytochemistry 67: 1406-1413.

Young, C.A.; Felitti, S.; Shields, K.; Spangenberg, G.; Johnson, R.D.; Bryan, G.T.; Saikia, S.; Scott, B. 2006. A complex gene cluster for indole-diterpene biosynthesis in the grass endophyte Neotyphodium lolii. Fungal Genetics and Biology 43: 679-693. 